\title{
Adult-onset presentation of cervicothoracic split cord malformation: illustrative case
}

\author{
Huei Ti Soh, MD \\ Department of Rehabilitation Medicine, Nepean Hospital, Sydney, New South Wales, Australia
}

BACKGROUND A 60-year-old female presented with a 6-month history of progressive lower limb pain, weakness, and declining mobility. She was initially diagnosed as having possible hip osteoarthritis or ligamental knee injury. She was eventually seen by a neurologist, who admitted her to a tertiary hospital with new-onset upper motor neuron signs and urinary incontinence. Magnetic resonance imaging of the whole spine revealed evidence of C7-T2 type 1 split cord malformation (SCM) necessitating urgent spinal surgery. She had an excellent outcome with intensive rehabilitation and returned to her premorbid level of function and mobility.

OBSERVATIONS The patient presented with nonspecific symptoms, which led to multiple referrals and a significant delay in her diagnosis.

LESSONS Clinicians should be aware of the importance of a detailed history with thorough neurological and spinal examinations.

https://thejns.org/doi/abs/10.3171/CASE21415

KEYWORDS diastematomyelia; split cord malformation; rehabilitation

The purpose of this article is to present an extremely rare case of adult-onset presentation of cervicothoracic split cord malformation (SCM). The importance of an appropriate clinical history and examination with thorough and effective communication between different specialties is highlighted.

\section{Illustrative Case}

A previously healthy 60-year-old female of Indian Fijian descent presented with a 6-month history of gradually progressive lower limb weakness and left lateral thigh pain radiating to the knee. Her mobility declined from being a community ambulator without aids to using a wheelchair for all mobility over the course of 6 months. She revealed a past medical history of spina bifida diagnosed at birth requiring surgical repair in infancy with no residual deficits only after she was diagnosed with SCM on the basis of recent whole-spine magnetic resonance imaging (MRI).

Her symptoms were initially managed as those of degenerative osteoarthritis. Progressive symptomatology led to an emergency department visit, where it was thought she had a suspected knee ligament injury. A review by the orthopedic surgeons ruled out this diagnosis, and they suggested a referral to a neurologist. Her symptoms continued to progress with worsening mobility followed by onset of spasticity and urinary incontinence. The neurologist recommended an urgent hospital admission for further investigation.

In her initial assessment, she had an unremarkable upper limb and truncal examination with weakness in the lower limbs (proximal weakness greater than distal weakness), hyperreflexia and spasticity in her left leg, and bilateral ankle clonus. MRI of her spine revealed evidence of partial fusion of the vertebral bodies with hydromyelia at T2 and T3 (Fig. 1) and type 1 SCM at the level of C7-T2 with spina bifida (Fig. 2).

She then underwent a C6-T1 laminectomy with decompression of the T1 intramedullary cyst. Postoperatively, the patient noticed immediate improvement in her lower limb strength and return of urinary continence.

Upon transfer to the rehabilitation ward, she had bilateral lower limb weakness, neuropathic pain in the left leg, reduced proprioception, and mild left leg spasticity. She required assistance to mobilize for short distances using a forearm support frame.

Over the next 3 weeks, she made consistent neurological and functional gains. Her leg spasticity and pain also significantly reduced.

ABBREVIATIONS CT = computed tomography; MRI = magnetic resonance imaging; $\mathrm{SCM}=$ split cord malformation . INCLUDE WHEN CITING Published September 27, 2021; DOI: 10.3171/CASE21415.

SUBMITTED August 9, 2021. ACCEPTED August 17, 2021.

(c) 2021 The authors, CC BY-NC-ND 4.0 (http://creativecommons.org/licenses/by-nc-nd/4.0/). 


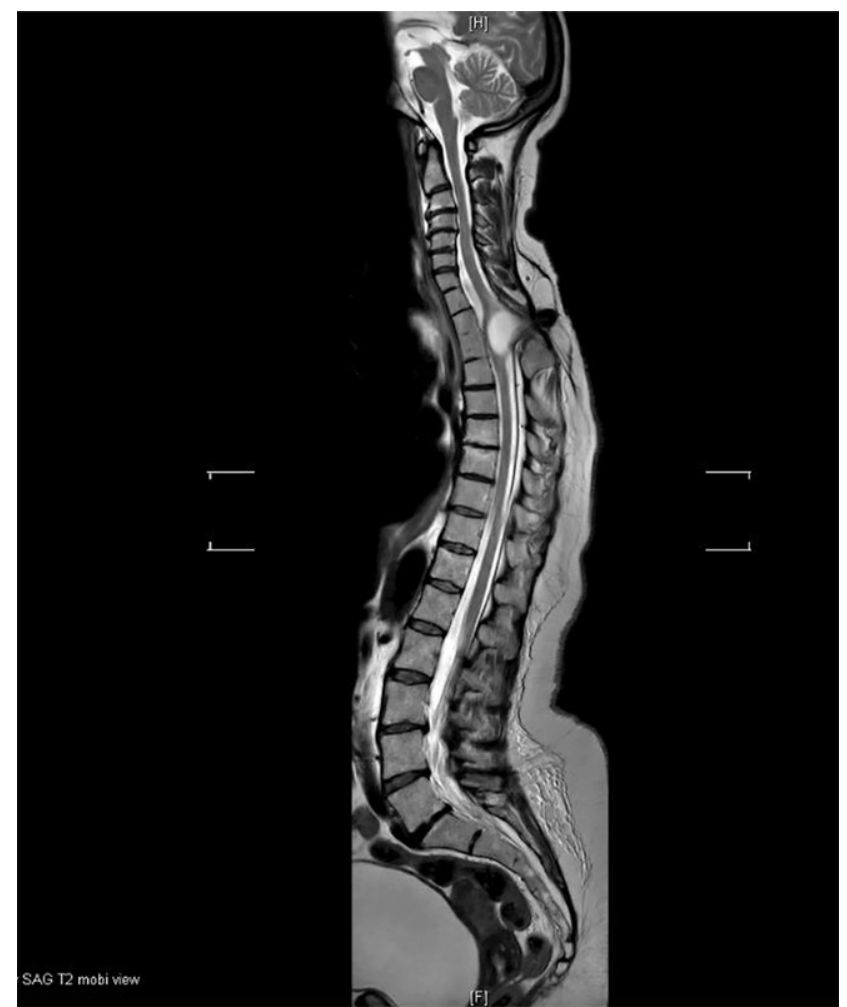

FIG. 1. Initial MRI scan showing sagittal plane with hydromyelia noted at T2-3 level.

She was walking independently with a four-wheeled walker upon discharge from inpatient rehabilitation and achieved premorbid baseline mobility (unaided community ambulation) after just 4 weeks of outpatient physiotherapy.

\section{Discussion}

SCM is a rare congenital disorder accounting for $4 \%$ of total spinal dysraphisms where the spinal cord is divided into two hemicords by an interposing septum. ${ }^{1}$ In type 1 SCM, also known as diastematomyelia, the two hemicords are contained within their own

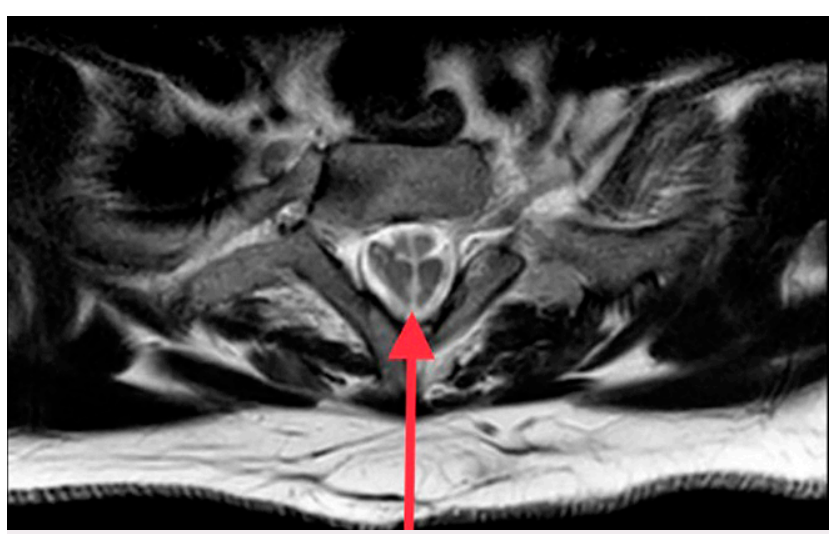

FIG. 2. Initial MRI scan showing axial plane with evidence of a type 1 SCM. Two hemicords, each contained within its own dural sac, are separated by a median bony spur (red arrow). dural sacs separated by a rigid osseocartilaginous septum. In type 2 SCM (also known as diplomyelia), the hemicords are contained within a single dural sac interposed by a nonrigid fibrous septum. Type 1 SCM causes more severe symptoms than type $2 .^{2}$

SCM has a female predominance of $70 \%-96 \%{ }^{3}$ These malformations are most commonly seen in the lumbar or lumbosacral region, followed by the thoracic, then the cervical, and very rarely the cervicothoracic spine. ${ }^{3-5}$ SCM is commonly associated with other spinal anomalies such as hydromyelia and meningomyelocele. Adult-onset presentations are rare because a vast majority of patients are diagnosed in infancy or early childhood with symptoms of tethered cord syndrome, association with other spinal dysraphisms, orthopedic deformities, or cutaneous lesions. ${ }^{2,4,6}$ In adults, the mean age range at time of diagnoses is $26-38$ years., ${ }^{3,7}$ With advances in modern imaging, adults are being diagnosed with increasing frequency.

Symptomatic patients typically present with signs and symptoms associated with tethered cord. ${ }^{2,8,9}$ These deficits arise from transfixation of the spinal cord by the dividing septum, which prevents the normal upward movement of the spinal cord during growth,, ${ }^{2,8}$ leading to ischemia and tissue necrosis at the level of the defect. ${ }^{2}$ Patients also frequently present with orthopedic deformities such as scoliosis and talipes, as well as cutaneous lesions such as hypertrichosis and hemangioma. ${ }^{2-6}$ In asymptomatic patients, particularly in the pediatric population, orthopedic and skin changes may be the only clue. 3,10

Plain radiographs can reveal clues to diagnosing SCM, including scoliosis, widening of interpeduncular distance, anteroposterior narrowing of vertebral bodies, and segmental vertebral anomalies. ${ }^{3}$ Computed tomography (CT) myelography or three-dimensional CT help differentiate type 1 and type 2 SCMs. MRI complements CT findings and is the modality of choice for detecting SCM., ${ }^{2,3,5}$ Urodynamic studies are an important tool to prevent renal deterioration because symptoms of neuropathic bladder changes may not be overt in some cases. ${ }^{11}$

Due to the progressive nature of this disease, most patients require surgical management involving laminectomy, untethering of the cord, and septum resection. In symptomatic patients who underwent operative management, studies reported significant improvement in neurological function. ${ }^{2,3,5,12}$ Postoperative complications were reported in a small proportion of patients, with few requiring reoperations secondary to regrowth of the disposing septum. Recommendations are conflicting for asymptomatic patients. Several studies advocate for prophylactic surgery, especially in pediatric cohorts $^{1,5,7}$ and in those with type 1 SCM on the basis that neurological deficits may be irreversible once they develop. ${ }^{1}$ Other studies recommend expectant management in those in whom the disorder remains asymptomatic.

\section{Observations}

Adult-onset presentation of symptomatic cervicothoracic SCM in an elderly patient is extremely rare.

\section{Lessons}

A missed or delayed diagnosis of SCM can lead to permanent neurological sequalae. Asymptomatic SCM is being detected more often due to advancements in imaging. SCM has the potential to be misdiagnosed due to its rarity. 


\section{Acknowledgments}

I acknowledge Dr. Agapito Nario, Dr. Taranpreet Bains, and Dr. Jee Hye Kim for their help and contribution to this article.

\section{References}

1. Viswanathan VK, Minnema AJ, Farhadi HF. Surgical management of adult type 1 split cord malformation. Report of two cases with literature review. J Clin Neurosci. 2018;52:119-121.

2. Cheng B, Li FT, Lin L. Diastematomyelia: a retrospective review of 138 patients. J Bone Joint Surg Br. 2012;94(3):365-372.

3. Ahmed AK, Howell EP, Harward S, et al. Split cord malformation in adults: literature review and classification. Clin Neurol Neurosurg. 2020;193:105733.

4. Huang S-L, He X-J, Wang K-Z, Lan B-S. Diastematomyelia: a 35-year experience. Spine (Phila Pa 1976). 2013;38(6):E344-E349.

5. Schijman E. Split spinal cord malformations: report of 22 cases and review of the literature. Childs Nerv Syst. 2003;19(2):96-103.

6. Erşahin Y. Split cord malformation types I and II: a personal series of 131 patients. Childs Nerv Syst. 2013;29(9):1515-1526.

7. Russell NA, Benoit BG, Joaquin AJ. Diastematomyelia in adults. A review. Pediatr Neurosurg. 1990-1991;16(4-5):252-257.

8. Pang D. Split cord malformation: Part II: Clinical syndrome. Neurosurgery. 1992;31(3):481-500.

9. Mahapatra AK. Split cord malformation - a study of 300 cases at AlIMS 1990-2006. J Pediatr Neurosci. 2011;6(3 Suppl 1):S41-S45.
10. Wenger M, Hauswirth CB, Brodhage RP. Undiagnosed adult diastematomyelia associated with neurological symptoms following spinal anaesthesia. Anaesthesia. 2001;56(8):764-767.

11. Proctor MR, Bauer SB, Scott RM. The effect of surgery for split spinal cord malformation on neurologic and urologic function. Pediatr Neurosurg. 2000;32(1):13-19.

12. Lee GY, Paradiso G, Tator CH, Gentili F, Massicotte EM, Fehlings MG. Surgical management of tethered cord syndrome in adults: indications, techniques, and long-term outcomes in 60 patients. J Neurosurg Spine. 2006;4(2):123-131.

\section{Disclosures}

The author reports no conflict of interest concerning the materials or methods used in this study or the findings specified in this paper.

\section{Supplemental Information}

Previous Presentations

This work has been presented previously in a prerecorded poster presentation at the Virtual Snapshot 2021 of the Rehabilitation Medicine Society of Australia and New Zealand on July 31, 2021.

\section{Correspondence}

Huei Ti Soh: Nepean Hospital, Sydney, New South Wales, Australia. hueiti.soh@health.nsw.gov.au. 